\title{
Chess players' intake of task-relevant cues
}

\author{
PERTTI SAARILUOMA \\ University of Helsinki, Helsinki, Finland
}

\begin{abstract}
Patterns of chess pieces are important for chess players in storing information in memory-recall tasks. It also may be assumed that these patterns play a central role in the perceptual coding of task-relevant cues. Two perceptual classification experiments were done to test this assumption. Chess-players' intake of task-relevant cues was studied by measuring the reaction times of subjects in counting the minor pieces on the chessboard or searching for checks in the game and in random positions. Surprisingly, there was no interaction between the level of skill and the type of position. In Experiment 3, both a perceptual classification task and a memory-recall task using the same positions were presented to a group of chess players at the same session. In the perceptual classification task, there was no interaction between the level of skill and the type of position, but in the recall task there was strong interaction. The result suggests that the information intake of chess players, in its lowest levels, is not dependent on the learned patterns. The role of the patterns becomes important in defining the information to be looked for, but the patterns are not important in the early-stage perceptual structuring of a position.
\end{abstract}

Human problem solving traditionally has been considered either in terms of memory or perception. On one hand, the associationists, such as the British empiricists, classical associationists, and behaviorists, have all emphasized the role of previously learned knowledge in human problem solving (Humphrey, 1951; Mandler \& Mandler, 1964). On the other hand, the Gestaltists, for example, have argued that perception rather than memory is a crucial cognitive function in human problem solving (e.g., Köhler, 1917/1957). They have argued that, because perceptual restructuring is essential for finding genuinely new solutions, it is the very essence of the problem solving process. The difference between the two theoretical approaches became evident in the "trial-anderror-versus-insight" discussion of the first half of this century.

The "perception-versus-memory" dispute dates back to at least the 17th century and Locke (1690/1961) and Leibniz (1704/1979). Recently, the associationists have had the upper hand. Modern cognitive theories of human problem solving very often have been basically associationistic. Until the 1980s there was little discussion about the possible role of perceptual functions in human problem solving, although the importance of the perceptual processes has long been recognized (see Chase $\&$ Simon, 1973; de Groot, 1966). One reason for this lack of discussion is that de Groot's famous experiments with chess players very strongly support the associationistic position. He showed that skilled chess players are much better than less skilled players in recalling chess positions after short

This study has been supported by The British Council and Academy of Finland. I thank Dr. Alan Allport for helpful discussions. Requests for reprints should be addressed to the author at the Department of Applied Psychology, University of Helsinki, Fabianinkatu 33, 00170 Helsinki 17 , Finland. presentations, when the presented positions have been taken from real chess games. In "random positions," in which the pieces have been scattered on the board, skilled players are not essentially better than their less skilled competitors (Chase \& Simon, 1973; de Groot, 1965, 1966).

These results mean that, over thousands of hours of practicing, skilled players acquire a vast store of chessspecific patterns of pieces, enabling them to code chess positions in chunks of a few pieces. The better a player, the larger the chunks he (she) is able to use in coding a position for recall. In random positions, however, he is unable to use his experience, as the pieces are scattered and do not form familiar configurations (Chase \& Simon, 1973; de Groot, 1966; Newell \& Simon, 1972).

Thus, learned patterns of pieces play a very important role in recall. However, they also perform a very important part in problem solving. Anyone having some experience with chess combinations knows that skilled players also use the patterns of chess pieces in searching for a move in problematic chess positions. A very important part of chess training is precisely the study of these properties of chess positions (Lasker, 1975). In addition, it can be easily observed in the protocols of "thinkingaloud" experiments that players use familiar patterns to find strong moves (de Groot, 1965).

Based on these previous experiments, it seems natural to assume that a chess player searching for a move works in a top-down manner. When he sees a chess position, it activates in his mind a group of typical patterns and moves associated to them. He simply selects from the various familiar patterns. Perception is not essential in this process. It is important only in adapting the incoming information, so that the problem of matching the learned patterns to the patterns in a problem position is possible to solve. 
De Groot's $(1965,1966)$ phenomenon is very general. It is valid for various games, such as "Go" (Reitman, 1976) and bridge (Charness, 1979). It has also been demonstrated in more realistic task environments: in music (Sloboda, 1976), in computer programming (Jeffries, Turner, Polson, \& Atwood, 1981; McKeithen, Reitman, Rueter, \& Hirtle, 1981), and in electronic technology (Egan \& Schwartz, 1979). As it is such a general phenomenon, it is natural to conclude that in one way or another the aspects of chess players' problem solving really can be generalized to the whole of human problem solving, which can be regarded basically as a process of associating learned knowledge in new problem situations (Chi, Glaser, \& Rees, 1982).

It is evident that there is much truth in this approach, but it is by no means self-evident that this picture of human problem solving is exhaustive. The experimental evidence supporting these ideas comes mostly from memoryrecall experiments. As a result, evidence naturally supports a model based on memory recall. In order to convince ourselves of this associationistic thinking, the relevance of chess-specific patterns should also be tested in perceptual tasks, which are relevant to chess players' problem solving.

One perceptual task that is clearly relevant to chess players' information processing is the perceptual classification of task-relevant cues. A chess player must be constantly aware of the positions of the pieces, of threats, of strategic and tactical formations, and so forth. These factors may be termed task-relevant cues. They are properties of a chess position that must be noticed by a player for him to be able to play. Hence, the intake of task-relevant cues is a necessary condition for rule-ordered chess.

When trying to find a move, a chess player constantly and automatically looks for task-relevant cues. If he misses a task-relevant cue, such as a threat, he very probably loses, and his problem solving process fails. Blunders, or severe tactical errors, are typical examples of this kind of incident. The more skilled a player is, the less often he blunders, but even very high-level grandmasters make occasional blunders (Krogius, 1976; Saariluoma, 1984). In fact, a surprisingly large number of chess games are decided by one of the players failing to detect task-relevant cues. This means, of course, that perceptual classification is a very relevant task in considering chess players' problem solving.

If the associationistic thinking arising from de Groot's $(1965,1966)$ phenomenon really is an exhaustive description of chess players' problem solving, there should be effects in perceptual classification tasks similar to those in memory-recall tasks. If, in reality, chess players' information processing is purely memory-controlled, there should be a strong interaction in perceptual tasks, as there is in memory-recall tasks. The skilled chess players should be much more skilled than the less skilled in game positions, but not essentially better in random positions.
To test this assumption, three experiments were carried out. In Experiment 1, subjects were asked to decide as fast as possible whether, in the presented position, one king was in check. In Experiment 2, the subjects' task was to count the number of minor pieces on the board as fast as possible. In Experiment 3, Experiment 2 was replicated with minor changes, but in the same session the same subjects also took part in de Groot's (1965) recall experiment.

\section{EXPERIMENT 1}

Threats are very important in the visual world of chess players. A chess player who fails to see that one of his pieces is en prise will probably lose the game. Thus, it is not surprising that the gaze of a chess player fixes on the attacking and defending pieces (Simon \& Barenfeld, 1969). In addition, it is impossible to play legal chess if a player does not see that his king is threatened by another piece (i.e., the king is in check).

Chess players quite often miss elementary threats; however, the more experienced a player is, the less often he makes blunders. In competitive chess, novices make blunders in every second game they play, while grandmasters do so only a few times throughout their grandmaster careers (Krogius, 1976; Saariluoma, 1984). It is just as important for a chess player to be aware of threats as it is for the player to notice other tactical or strategic features in a position. Threats are thus typical task-relevant cues.

The first experiment was designed to explore the detection of elementary threats. As there are usually several different threats in an ordinary game position, it was necessary to specify the target more precisely and to select a specific kind of threat, check, as target. It should be noted that there is nothing exceptional in selecting check as a target, because positions in which a player checks are quite ordinary to chess games.

In accordance to the main hypothesis, check detection was studied in game and random positions. If skilled chess players do benefit from their learned patterns of pieces in the same way as they do in memory experiments, there should be strong interaction between the game and random positions, so that skilled players prove to be much better than the less skilled in game but not in random positions.

\section{Method}

Subjects. Fourteen male chess players participated in the experiment as volunteer subjects. Their ages ranged from 12 to 55 years. The subjects were divided into three skill groups using the Finnish SELO gradings. SELO grading points are equivalent to the international ELO grading points, which have been explained in detail by Elo (1978). The Masters Group consisted of 3 players with more than 2,000 SELO points. Two of them had achieved the master norm, that is 2,200 SELO points. The Second Class had 5 players with gradings between 1,701 and 2,000 points. In the Beginners Group, there were 6 players with 1,700 points or less. 
Design and Materials. Using chess print transfers, 120 positions with 23-24 pieces in each were made. Half were game positions and half were made by randomizing the background pieces, leaving the king and the checking piece unmoved. One of the kings was in check in half of the positions. All positions had been taken from Neistadt's Shakmatnyj Praktikum (1980), which at the time of the experiment had not been translated into English or German. so the subjects were not familiar with the book. The positions were photographed and made into slides.

The slides were presented in a series. The task of the subjects was to say, as fast as possible, whether either of the kings was in check. Each slide was shown for $10 \mathrm{sec}$, independent of the subjects' reaction. The pause between two successive slides was $1.5 \mathrm{sec}$. There was a break of approximately $1 \mathrm{~min}$ after every 30 slides while the magazine of the projector was changed and the subjects were asked about their personal chess history.

The slides were shown with a slide projector, which had a timer and a shutter. The display size was $40 \times 40 \mathrm{~cm}$, and the subjects sat at a distance of $150 \mathrm{~cm}$ from the screen. The midpoint of the chessboard was $100 \mathrm{~cm}$ from the surface of the floor. The vocal yes/no reaction times were collected. The room was lighted and the screen was white.

\section{Results}

The means for each skill group were calculated for four different conditions: game and check $(\mathrm{G}+)$, game and no check $\left(\mathrm{G}^{-}\right)$, random and check $(\mathrm{R}+)$, and random and no check $(\mathbf{R}-)$. The results are summarized in Figure 1 , which includes the mean reaction times of the three skill

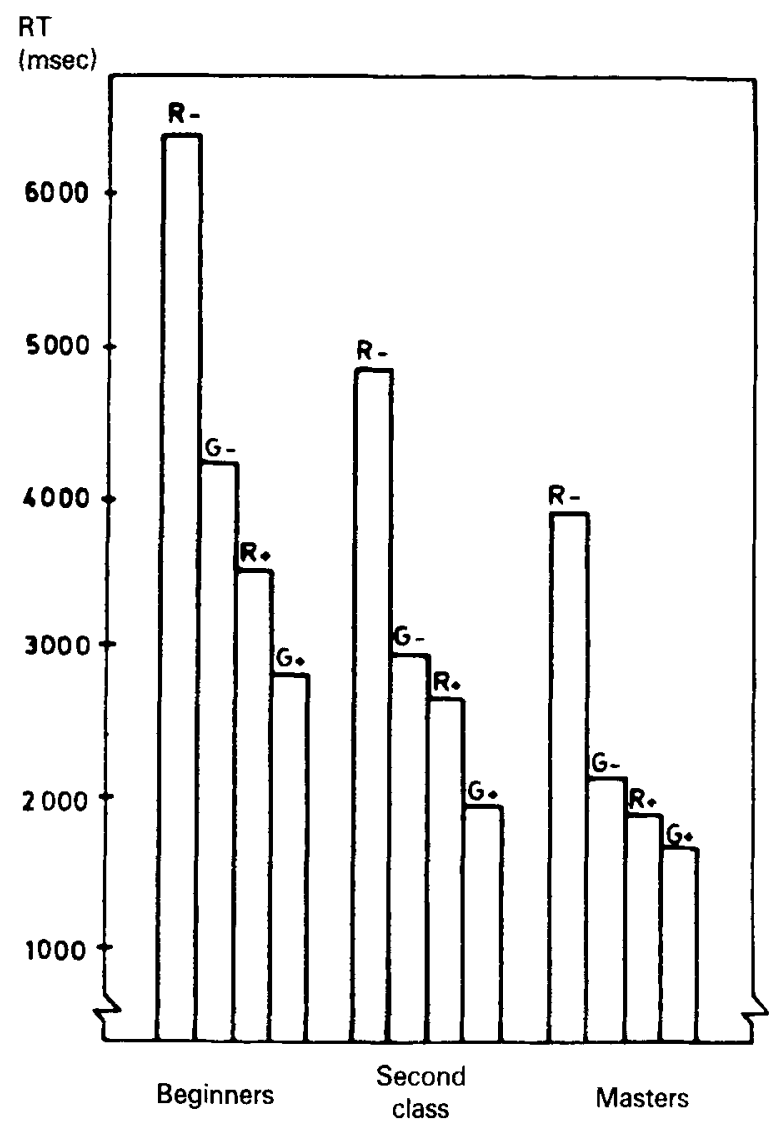

Figure 1. Reaction times of the subjects at three skill levels in four different conditions: game and check $(G+)$, game and no check ( $G-)$, random and check $(R+)$, and random and no check $(R-)$.
Table 1

Error Distribution in Check-Detection Task

\begin{tabular}{lccc} 
& \multicolumn{3}{c}{ Level of Skill } \\
\cline { 2 - 4 } & Beginners & Second Class & Masters \\
\hline Game $(+)$ Positions & $8 \%$ & $3 \%$ & $2 \%$ \\
Random $(+)$ Positions & $13 \%$ & $13 \%$ & $4 \%$ \\
\hline
\end{tabular}

groups. The main effects of skill $[\mathrm{F}(2,11)=10.41$, $\mathrm{p}<.01]$ and type of position [i.e., game vs. random, $\mathrm{F}(1,11)=115.06, \mathrm{p}<.001]$ and the type of response [i.e., check vs. no check, $F(1,11)=282.38$, $p<.001$ ] were all significant. All the interactions, with the exception of the type of position versus the type of response $[F(1,11)=19.95, p<.001]$, were insignificant. The error distribution is presented in Table 1. All the errors were errors of omission. There were more errors in random than in game conditions, and the skilled players made fewer errors than the less skilled ones, in both game and random conditions. The effect of skill on the number of errors $[\mathrm{F}(2,11)=1.99]$ was not significant, but the effects of randomization $[F(2,11)=82.40, p<.001]$ and the interaction type of position versus the level of skill $[F(2,11)=6.30, p<.05]$ were significant. This timeerror pattern bears some resemblance to the reaction-time distribution.

\section{Discussion}

The skilled players were clearly faster than the less skilled in the check-detection task. This was to be expected, as it is well known that practice increases speed in perceptual classification tasks, and it is probable that skilled players have had more practice than less skilled ones. Analogous findings have been presented in many perceptual classification tasks (Neisser, 1963; Neisser, Novik, \& Lazar, 1963; Rabbitt, 1978; Rabbitt, Cummings, \& Vyas, 1979).

The results show a total absence of the expected interaction between skill and type of position in reaction time. The skilled players were clearly faster in random as well as in game conditions. This result is contrary to the hypothesis and indicates that the role of chunks in perception is not the same as in memory recall. If it were, interaction in this task should be similar to that in de Groot's $(1965,1966)$ experiments. Another aspect of the results is that the difference between the two conditions is practically additive. The random positions were proportionately as difficult for the skilled players as for the less skilled. In addition, all subjects used more time to say no in random conditions than in game conditions. This is also contrary to the main hypothesis. If the skilled players used their previously learned patterns of pieces, then there should have been strong interaction in reaction times between game and random positions, but there was an additive difference between them.

One may argue that the error patterns showed some kind of interaction. The difference in errors between the game and the random positions in this experiment is not additive. The difference is clearly smaller for skilled than for 
less skilled players. This fact contradicts the main hypothesis. In memory-recall experiments the difference between game and random positions is greater for skilled than for less skilled players, which also is in contradiction to the main hypothesis.

\section{EXPERIMENT 2}

Before formulating any further conclusions, it is necessary to see whether the results of Experiment 1 may be generalized in other kinds of perceptual classification tasks. It may be that the result pattern of the previous experiment holds only for check detection. It is known that in chess-recognition memory there is no interaction between the type of position and the level of skill when study time is unlimited (Goldin, 1979). When study time is limited, however, an additive difference arises between the two types of position, independent of the level of skill, similar to that in the previous reaction-time experiment (Saariluoma, 1984). The familiarity of the target might be one important factor in perceptual classification; therefore, it might be reasonable to change the target so that it is in no way a familiar piece configuration. Although there is a huge number of possible checks if the locations of the checking pieces and kings are included, there are only five different checks for one side if the locations are excluded. This means that check, as a pattern and target, may be very familiar, and it is advisable to try to construct a perceptual classification task in which the target is less familiar.

A less familiar target is the configuration of minor pieces, which can be used as target in the minor piececounting task. In this task, subjects must say as fast as possible how many minor pieces, that is, bishops and knights, there are in a position. The locations of the minor pieces are quite unpredictable (de Groot, 1966) and so their configurations are still more unpredictable. This task should decrease the effects of target familiarity. The pieces are, of course, more familiar to skilled players, but the absolute effect of piece recognition on reaction-time differences is small (Saariluoma, 1984). The configurations vary so much that targets in this task cannot be very familiar to the subjects.

\section{Method}

Subjects. Six subjects participated in Experiment 2. Two of them were master-level players with English gradings corresponding to approximately 2,350 SELO points. Two of the subjects were firstand second-class players with English gradings which corresponded on average to approximately 1,880 SELO points. Two of the subjects were from a subject panel with no grading. They were paid f1.25 per hour for participating in the experiment. The subjects were grouped as above.

Design and Materials. One-hundred twenty slides were prepared as in the previous experiment. In each position there were 20-25 pieces; half were game positions and half random. There were from zero to eight minor pieces in each. The random positions were made by scattering all the pieces on the board so that the locations of the target pieces were not the same in the game and random positions. The positions were taken from various chess periodicals.
Table 2

Error Distribution in Minor Piece-Counting Task

\begin{tabular}{lccc} 
& \multicolumn{3}{c}{ Level of Skill } \\
\cline { 2 - 4 } & Beginners & Second Class & Masters \\
\hline Game Positions & $7 \%$ & $5 \%$ & $5 \%$ \\
Random Positions & $15 \%$ & $12 \%$ & $8 \%$ \\
\hline
\end{tabular}

The apparatus and overall organization were the same as in Experiment 1 . The slides were presented in a series, but each slide was changed immediately after the subjects' reaction. There was a pause of $3.5 \mathrm{sec}$ between each reaction and the successive slide. The screen was dark during the pause. In the middle of the experiment, there was a pause of approximately $1 \mathrm{~min}$, during which the magazine of the projector was changed.

\section{Results}

The results of the experiment are summarized in Figure 2. This includes the means for the three different skill groups. The effect of skill $[\mathrm{F}(2,3)=43.17, \mathrm{p}<.01]$ and the type of position $[\mathrm{F}(1,3)=199.8, \mathrm{p}<.001]$ were significant. The interaction of skill and the type of position, however, was not significant $[\mathrm{F}(2,3)=4.71]$.

The percentage of errors (Table 2) was relatively high in all the skill groups. The percentage of errors was largest in the beginners group, but there was no strong skill effect. All the subjects made more errors in random than in game conditions. The effects of skill $[\mathrm{F}(2,3)=.18]$

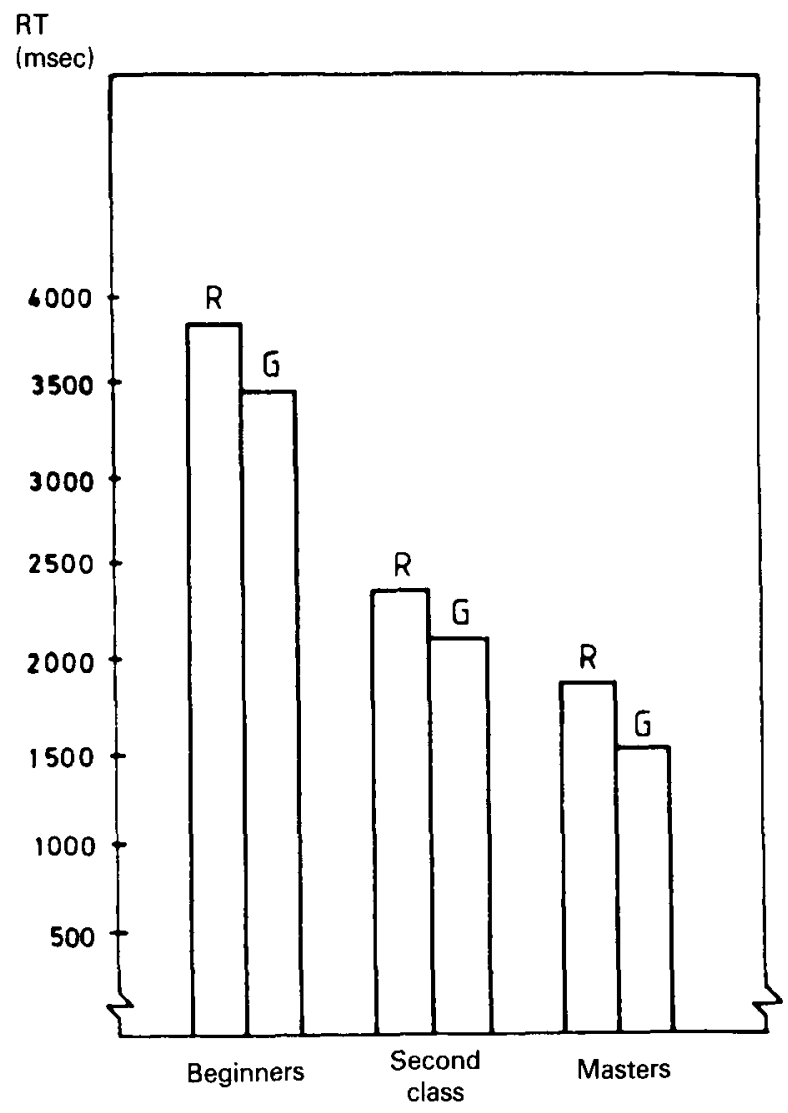

Figure 2. Reaction times of three skill groups in minor piece counting task, in game (G) and random $(R)$ positions. 
and the interaction of skill and the type of position $[\mathrm{F}(2,3)$ $=.88$ ] were not significant, but the effect of the type of position $[F(1,3)=14.8, p<.05]$ was significant.

\section{Discussion}

The skill difference was again very clear. The more skilled players were clearly faster than the less skilled. Again, this is to be expected. The skilled players have seen chess positions far more frequently and, therefore, are faster in chess-specific perceptual classification tasks.

In this experiment, there was also an additive difference between the two types of positions. The skilled players were faster in game as well as in random positions. The difference between the types of positions is skill-independent. Random positions proved more difficult than game positions for all subjects, in equal proportions.

The error pattern was similar to that obtained in Experiment 1 . The skilled players made fewer errors than the others in game and in random conditions. The random positions were more difficult for all of the subjects. The difference between game and random positions again was smaller in skilled players than in less skilled players. However, this does not support the main hypothesis. In de Groot's $(1965,1966)$ experiment, the pattern of results was the opposite.

Experiment 2, like Experiment 1, is in contradiction to the main hypothesis. Greater skill does not cause any interaction between the two types of position. The familiarity of the target pattern is also insignificant in this respect. The only possible way to save the main hypothesis is to argue that these effects depend somehow on the nature of the subjects or on the nature of the positions. It may be that the skill difference, in spite of its being more than 1,000 SELO points or approximately five standard deviations, is nevertheless not large enough.

\section{EXPERIMENT 3}

Experiment 3 is the crucial one. It was designed to demonstrate that de Groot's $(1965,1966)$ phenomenon and the perceptual classification of chess positions are qualitatively different in respect to the interaction between skill and the type of positions. Naturally, this would mean that the main hypothesis is wrong, and that chess players do not use their learned patterns in coding a chess position for the intake of task-relevant cues.

This experiment consisted of two tasks. The first was a perceptual classification task, which was basically the same as in Experiment 2. The second task was de Groot's (1965) memory-recall task. Both tasks were shown to the same subjects during the same experimental session, and the positions in de Groot's task were taken from those used in the first task.

\section{Method}

Subjects. Nine subjects participated in the experiment. Three of them were master-level players with more than 2,200 SELO points. One of them was a strong international master. Three of the sub- jects were third- to second-class players with an average SELO grading of approximately 1,800 SELO points. Another 3 subjects were beginners with an average SELO grading of approximately 1,400 SELO points. The ages of the subjects ranged from 16 to 25 years, with the exception of 1 subject who was 37 . The subjects were divided into groups as above. They were paid 50 FMK per hour.

Materials and Design. One-hundred twenty positions with 20-28 pieces were prepared on slides as before. When randomizing the positions, which were taken from various chess periodicals and a book of combinations, the minor pieces were left intact and only the background pieces were randomized.

A perceptual classification experiment was carried out in a similar way to the previous two experiments, with the exception that subjects were strongly instructed to avoid errors. In the recall experiment, the slides were shown for $5 \mathrm{sec}$ each, and the subjects recalled the positions immediately after the presentation. The recalled positions were the first 20 positions presented in the perceptual classification experiment. Half were game positions and half were random.

All subjects took part first in the perceptual classification experiment, and immediately afterward they participated in the recall experiment. The session lasted approximately $1 \mathrm{~h}$.

\section{Results}

The results of Experiment 3 are summarized in Figure 3. The left side of Figure 3 presents the results of the minor piece-counting task and on the right are the results of the memory-recall task. The results are very much what one might expect. The perceptual classification results are very similar to those in Experiment 2, and the results of the memory-recall task replicate de Groot's $(1965,1966)$ results.

The effects of skill $[F(2,6)=40.29, p<.001]$ and the type of position $[F(1,6)=59.23, p<.001]$ are significant in the perceptual classification task. The interaction between the main effects $[F(2,6)=1.9]$ is not significant. In the recall task, the main effects of skill $[F(2,6)$ $=31.04, \mathrm{p}<.001]$ and of type of position $[\mathrm{F}(1,6)=$ $124.15, \mathrm{p}<.001]$ are significant. In addition, the interaction of the main effects is also significant $[F(2,6)=$ $15.23, \mathrm{p}<.01]$.

The error distribution (see Table 3 ) was very similar to that in the second experiment, although the subjects were instructed to avoid errors. The most skilled players made fewer errors than the second-class players, but the beginners were quite careful this time. None of the effects was significant.

\section{Discussion}

This experiment again contradicts the main hypothesis. There was no interaction in the perceptual classification task, but the interaction was strong in the memory-recall task. It is evident that, in perceptual classification tasks, the effect of randomization is different from the effect in memory-recall tasks. This means that the main hypothesis must be abandoned.

\section{GENERAL DISCUSSION}

There are three important findings in the perceptual classification experiments. First, there was a clear skill 


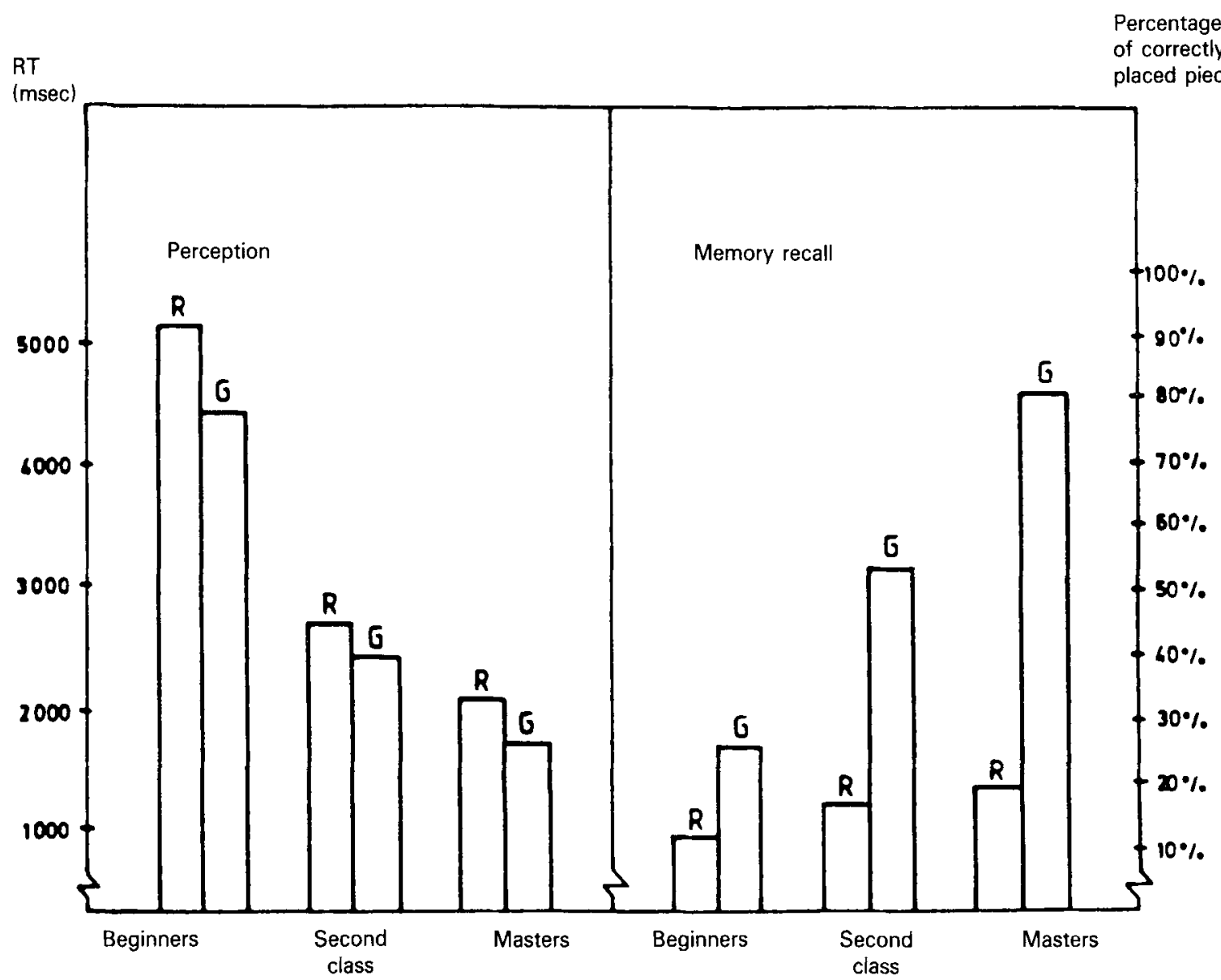

Figure 3. Reaction times of the subjects in minor piece counting task (left) and the percentage of correctly placed pieces in de Groot's (1965) recall task (right).

difference in the speed of perceptual classification. The skilled players were faster than the less skilled. Second, the random positions were more difficult than the game positions, and all the players were slower in random conditions. Third, the difference between the game and random conditions was additive, and there was no interaction between the level of skill and the type of position.

The first of the three findings is the easiest to explain. Skilled players are more familiar with chess-specific material than are less skilled players. The former have seen chess positions far more often, which helps to make them faster in processing perceptually chess-specific material. This is a well-known phenomenon and it has been demonstrated many times in different types of perceptual classification experiments (Neisser, 1963; Neisser,

Table 3

Error Distribution in Perceptual Classification Subtask

\begin{tabular}{lccc}
\hline & \multicolumn{3}{c}{ Level of Skill } \\
\cline { 2 - 4 } & Beginners & Second Class & Masters \\
\hline Game Positions & $6 \%$ & $5 \%$ & $4 \%$ \\
Random Positions & $5 \%$ & $10 \%$ & $5 \%$ \\
\hline
\end{tabular}

Novik, \& Lazar, 1963; Rabbitt, 1978; Rabbitt, Cummings, \& Vyas, 1979; Schneider \& Shiffrin, 1977).

The second finding is also understandable. Random positions are more difficult than game positions because their structure is broken. Normal chess positions are more or less harmonious. They have, to a large extent, unbroken pawn chains and relatively systematically organized systems of empty squares, which presumably are helpful in perceptual processing. Random positions lack such properties. It is known that such violated structures are more difficult to process in perceptual tasks. Wordsuperiority effects are one example (Rabbitt, 1978). Violated real-world scenes provide another (Biederman, 1981). When a "normal" structure of the figure is violated, it very often becomes less open to perceptual processing. Random positions constitute this kind of violated structure, so it is expected that their processing takes more time.

The third finding is more puzzling, and I am not going to suggest any exhaustive answer. The difference in reaction times between game and random positions is skillindependent. It is presumably caused by some objective properties of random positions compared to game posi- 
tions, and it has something to do with some task-necessary but not content-specific processes. Their specification is a future research problem.

The most important implication of the qualitative difference between intake of task-relevant cues and recall is that memory-driven models of human problem solving are not sufficient. The intake of task-relevant cues is necessary for reasonable problem solving in chess. If a player misses an important task-relevant cue, he is no longer able to solve a problem properly. It is necessary to see all the task-relevant cues. As the intake of task-relevant cues is necessary for chess players' problem solving, it is also necessary to take into account the qualitative difference between perceptual and memory tasks when considering chess players' problem solving.

Thus, chess players' problem solving is not simply a process of activating previously learned patterns and the moves associated with them; it also is necessary to consider the problems of perceptual organization. This was suggested more than a decade ago by Chase and Simon (1973), when they postulated a perceptual preprocessing system called the "mind's eye" (see also Newell, 1972). The perceptual classification experiments suggest that one of the main functions of this system is to separate the target from the background.

It is evident that chess-specific patterns are important in chess players' problem solving; however, the patterns do not have much influence on information intake. It may be suggested that chess-specific patterns are important in defining targets for the attentional system, but that they do not play any role in the early perceptual structuring systems which organize the problem position into figure and ground. It may be that there are no skill-dependent differences in these systems, but that the objective characteristics of the stimuli are decisive.

\section{REFERENCES}

Biederman, I. (1981). On the semantics of a glance at a scene. In M. Kubovy \& J. Pomerantz (Eds.), Perceptual organization. Hillsdale, NJ: Erlbaum.

Charness, N. (1979). Components of skill in bridge. Canadian Journal of Psychology, 33, 1-16.

Chase, W., \& Simon, H. (1973). The mind's eye in chess. In W. Chase (Ed.), Visual information processing. New York: Academic Press.

Chi, M., Glaser, R., \& ReEs, E. (1982). Expertise in problem solving. In R. Stemberg (Ed.), Advances in the psychology of human intelligence (Vol. 1). Hillsdale, NJ: Erlbaum.

DE Groot, A. (1965). Thought and choice in chess. The Hague: Mouton. DE Groot, A. (1966). Perception and memory versus thought: Some old ideas and recent findings. In B. Kleinmuntz (Ed.), Problem solving. New York: Wiley.

Egan, D., \& Schwartz, B. (1979). Chunking in recall of symbolic drawings. Memory \& Cognition, 7, 149-158.

ELo, A. (1978). The rating of chessplayers, past and present. London: Batsford

GoLDIN, S. (1979). Recognition memory for chess positions: Some preliminary research. American Journal of Psychology, 92, 19-31.

HumphreY, G. (1951). Thinking. New York: Wiley.

Jeffries, R., Turner, A., Polson, P., \& Atwood, M. (1981). Processes involved in designing software. In $J, R$. Anderson (Ed.), Cognitive skills and their acquisition. Hillsdale, NJ: Erlbaum.

KöHLER, W. (1957). The mentality of apes. Harmondsworth: Penguin Books. (Original work published in 1917)

Krogrus, N. (1976). Psychology in chess. London: Pitman.

LASKER, E. (1975). Lasker's manual of chess. New York: Dover

LeIBNIZ, G. (1979). New essays on human understanding. Cambridge: Cambridge University Press. (Original work finished in 1704)

LOCKE, J. (1961). An essay concerning human understanding. London: Dent. (Original work published in 1690)

Mandler, J., \& Mandler, G. (1964). Thinking: From association to gestalt. New York: Wiley.

MCKeithen, K., Reitman, J., Rueter, H., \& Hirtle, S. (1981). Knowledge organization and skilled differences in computer program mers. Cognitive Psychology, 13, 307-325.

Neisser, U. (1963). Decision time without reaction time: Experiments in visual scanning. American Journal of Psychology, 76, 376-385.

Neisser, U., Novik, R., \& Lazar, R. (1963). Searching for ten targets simultaneously. Perceptual \& Motor Skills, 17, 955-961.

NEISTADT, J. (1980). Shakmatnyj praktikum. Moscow: Fiskultura i Sport.

NEWELL, A. (1972). A theoretical exploration of mechanisms for coding the stimulus. In A. Melton \& E. Martin, Coding processes in human memory. Hillsdale, NJ: Erlbaum.

Newell, A., \& Simon, H. (1972). Human problem solving. Englewood Cliffs, NJ: Prentice-Hall.

RabBitt, P. (1978). Sorting, categorization and visual search. In E Carterette \& M. Friedman (Eds.), Handbook of perception (Vol. 9) New York: Academic Press.

Rabbitt, P., Cummings, G., \& Vyas, S. (1979). Improvement, learning, and retention of skill at visual search. Quarterly Journal of $E x$ perimental Psychology, 31, 441-459.

ReITMAN, J. (1976). Skilled perception in Go: Deducing memory structures from inter-response times. Cognitive Psychology, 8, 336-356.

SaAriluoma, P. (1984). Coding problem spaces in chess: A psychological study. Commentationes scientiarum socialium 23. Turku: Societas Scientiarum Fennica.

Schneider, W., \& Shiffrin, R. (1977). Controlled and automatic in formation processing: Detection, search and attention. Psychologi cal Review, 84, 1-66.

SIMON, H., \& BARENFELD, M. (1969). Information-processing analysis of perceptual processes in problem solving. Psychological Review, 76, 473-483.

SLOBODA, J. (1976). Visual perception of musical notation: Registering pitch symbols in memory. Quarterly Journal of Experimental Psy. chology, 28, 1-16

(Manuscript received July 2, 1984; revision accepted for publication May 1, 1985.) 